\title{
DIE DENATIONALISIERUNG DES PRIVATRECHTS IN EUROPA - EINE GRUNDLAGEN- UND URSACHENANALYSE
}

\author{
The Denationalisation of Private Law in Europe - An Analysis of the \\ Fundamentals and Causes
}

\section{Dr. Hannes RÖSLER*}

I. Einführung, II. Europäische Union, 1. Weltgeschichtlich singuläre Souveränitätsabgabe, 2. Genese einer Gemeinschaft des Friedens, der Freiheit und der Wohlfahrt, 3. Wandelungen im Europaverständnis, III. Andere Entgrenzungsbemühungen als solche durch die EU, 1. Weitere Institutionen, 2. Außergemeinschaftlich initiiertes Privatrecht, a) Überblick zum wirtschaftsrelevanten Privatrecht, b) UN-Kaufrecht, IV. Ursachen, V. Gesamtbetrachtung

\section{ÖZET}

$\mathrm{Bu}$ makalede, Avrupa'daki ulusal özel hukukların uluslararasılaşmasının arkasındaki nedenler ve güçler incelenmiştir. Çalışmada, Avrupa Topluluğu özel hukukuna, ana mevzuatı ile birlikte, direktiflere ve Malların Uluslararası Satım Sözleşmeleri Hakkındaki Birleşmiş Milletler Sözleşmesi'ne (CISG) yer verilmiştir. Makale, Ole Lando'nun yardımıyla Avrupa Sözleşmeler Hukuku Komisyonu tarafından

* LL.M. (Harvard), Assessor. Wiss. Referent (Senior Research Fellow), Max-Planck-Institut für ausländisches und internationales Privatrecht (Max Planck Institute for Comparative and International Private Law), Hamburg, Deutschland. Der Artikel greift teils auf einen im European Legal Forum (EuLF) 2003, 207 (deutsch) bzw. EuLF 2003, 205 (englisch) erschienenen Beitrag zurück. 
geliştirilen “Avrupa Sözleşmeler Hukuku Prensipleri”ni ve UNIDROIT'da Michael Joachim Bonell tarafindan yönetilen uluslararası bir grubun yayımladığı "Uluslararası Ticari Sözleşmeler Prensipleri"ni konu edinmiştir. Bu çalışmayla, özel hukuk ile kamu hukuku arasındaki ayrımın uluslararası hukuk ve ekonomi tarafindan desteklenen ve giderek artan delilleri gösterilmeye çalışılmıştır. Bu süreç sadece Avrupa'ya münhasır değildir; ancak Avrupa Birliği'nin politik kurumları ve Avrupa Adalet Divanı yargilaması nedeniyle en fazla Avrupa'da hissedilmektedir. Üye devletlerin egemenlik yetkilerini AB'ye devretmeleri benzersiz bir olaydır. Avrupa Topluluğu paradigması, "bağımsız devletler federasyonu" nosyonuna uygun olarak, ticari borç senedi yaratma yetkisini kullanarak oluşturulmuş ve geliştirilmiştir. Bu yeni işbirliği modeli, egemenlik kaybına, çok taraflılığa, devlet faizlerine ilişkin davalarda özel hukuk kurumlarına üstünlük tanınmasına ve yüksek refah önceliğine neden olmuştur.

Anahtar kelimeler: Topluluk hukuku, CISG, Malların Uluslararası Satım Sözleşmeleri Hakkında Birleşmiş Milletler Sözleşmesi, Avrupa Adalet Divan1, Avrupa Özel Hukuku, lex mercatoria

\section{ABSTRACT}

The present article analyses the phenomena and forces behind the internationalisation of Europe's national private laws. It covers European Community private law with its main instrument, directives, and the internationally successful United Nations Convention on Contracts for the International Sale of Goods (CISG). As far as non-binding academic instruments are concerned, the article deals with the "Principles of European Contract Law" developed by the Commission on European Contract Law under the auspices of Ole Lando, as well as with the "Principles of International Commercial Contracts" issued by an international group led by Michael Joachim Bonell at UNIDROIT. The article seeks to show that there is an increasing evidence of a separation of state and private law, which is supported by international law and economics. This process is not limited to Europe as such, although the denationalisation of private law - through the European Union's political institutions and the judicature of the European Court of Justice - is most noticeable here. The transfer of Member States' sovereign powers to the EU is unprecedented. The paradigm of the European Community was formed and developed in keeping with the notion of a "federation of free states" using the power of trade in creating bonds, just like Immanuel Kant has advanced in "Toward Perpetual Peace" (1795). This new model of cooperation is marked by a loss of sovereignty, multilateralism, the preference for civil instruments in the pursuit of state interests and the primacy of increased prosperity.
\end{abstract}

Keywords: Community Law, CISG (United Nations Convention on 
Contracts for the International Sale of Goods), European Court of Justice, European Private Law, lex mercatoria.

\section{Einführung}

Weltweit und institutionell übergreifend mehren sich die Anhaltspunkte für eine stärkere Aufspaltung von Staat und Recht. Die damit verknüpfte Neuausrichtung des Privatrechts lässt sich nur durch den Prozess einer „Entmonopolisierung des Nationalen“1 im wirtschaftlichen, politischen und sozialen Leben erklären. Diese völkerrechtlich und ökonomisch unterstützte Entnationalisierung des Rechts wird nachfolgend einer evolutionärmethodischen und begründenden Untersuchung unterzogen. Hierbei wird insbesondere die Polyzentrizität und Polykontextualität des Privatrechts in Europa berücksichtigt, also das nationale Zivilrecht, das Gemeinschaftsprivatrecht, die beiden privatrechtlichen Prinzipienwerke und das UN-Kaufrecht in einer Zusammenschau analysiert.

\section{Europäische Union}

\section{Weltgeschichtlich singuläre Souveränitätsabgabe}

Die Europäische Union (EU) ist auf dem Weg zu einem gemeinsamen Zivilrecht und in vielen Bereichen ist die Europäische Rechtsgemeinschaft schon Realität: Etwa $80 \%$ aller deutschen Gesetze im Bereich des Wirtschaftsrechts sind durch das Gemeinschaftsrecht festgelegt, fast $50 \%$ aller deutschen Regelungen finden ihre Veranlassung im Gemeinschaftsrecht. ${ }^{2}$ Umfasst sind beispielsweise das Kauf-, Verbraucher-, Arbeits-, Dienst-, Werk-, Versicherungs- und Kreditrecht. ${ }^{3}$ Der Bestand an europäischen Rechtsregeln ist derart angewachsen, dass bereits weit über $50 \%$ des unionsweiten Bruttosozialprodukts - also einschließlich der inländischen Transaktionen - in gemeinschaftsrechtlich mitgeregelten Verträgen erwirtschaftet wird. ${ }^{4}$ Niemals zuvor hat es in Europa einen derart weitgehenden friedlichen und freiwilligen Zusammenhalt gegeben, wie er

1 W. Schäfer, Globalisierung: Entmonopolisierung des Nationalen?, in: H. Berg (Hrsg.), Globalisierung der Wirtschaft: Ursachen - Formen - Konsequenzen, (Schriften des Vereins für Socialpolitik, Neue Folge, Bd. 263), Berlin 1999, S. 9; s. auch Basedow/Kono (Hrsg.), Legal Aspects of Globalization, The Hague u.a. 2000.

2 So bereits erwähnt durch das BVerfG in seiner grundlegenden Maastricht-Entscheidung v. 12. 10. 1993, BVerfGE 89, 155 (173).

3 Zur Einführung in das Europäische Schuldvertragsrecht z.B. Basedow, Grundlagen des europäischen Privatrechts, JuS 2004, 89; Heiderhoff, Gemeinschaftsprivatrecht, München 2005; speziell zum Verbraucherrecht Rösler, Europäisches Konsumentenvertragsrecht Grundkonzeption, Prinzipien und Fortentwicklung, München 2004; Reich/Micklitz, Europäisches Verbraucherrecht, 4. Aufl., Baden-Baden 2003.

4 Grundmann, Europäisches Schuldvertragsrecht - Struktur und Bestand, NJW 2000, 14 (15). 
sich beständig seit Gründung der Europäischen Wirtschaftsgemeinschaft $(E W G)^{5}$ im Jahre 1957 in Breite und Tiefe entwickelt hat. Frühere - zumeist temporäre - Zusammenschlüsse beruhten demgegenüber regelmäßig auf Eroberung, Heiratspolitik oder gemeinsamer Ausbildung der Eliten. ${ }^{6}$

Das Besondere der neuen Zusammenarbeit besteht in der freiwilligen Übergabe von staatlicher Souveränität. ${ }^{7}$ Die Europäischen Institutionen können - so war es bereits bei der Europäischen Gemeinschaft für Kohle und Stahl aus dem Jahre 1951 (EGKS, kurz: Montanunion) angelegt - selbst Beschlüsse fassen und ihre Handlungen werden nicht von nationalen, sondern allein von dem Europäischen Gerichtshof (EuGH) in Luxemburg überprüft. ${ }^{8}$ Die Übergabe von hoheitlichen Rechten kommt auch noch in einem anderen Umstand zum Ausdruck: Anders als bei völkerrechtlichen Übereinkommen oder IPR-rechtlichen Regelungen kann etwa eine EGRichtlinie (Art. 249 EG-Vertrag) nicht mittels einseitiger Kündigung oder innerstaatlicher Maßnahme aus der Welt geschafft werden. ${ }^{9}$ Umgekehrt haben insbesondere die wirtschaftlichen Freiheiten des EG-Vertrages die Qualität subjektiver Rechte, deren Inhalte häufig erst durch einen gerichtlichen Entdeckungsprozess präzisiert werden. ${ }^{10}$

\section{Genese einer Gemeinschaft des Friedens, der Freiheit und der Wohlfahrt}

Der Impetus der selbstgewollten und breit angelegten Einigung durch die Kraft des Rechts lag zunächst weniger im Elan einer europäischen Bewegung, sondern - angesichts von zwei hier entflammten Weltkriegen und der Rohheit des Faschismus - in der gewandelten Rolle der Nation. Die Gegner von einst wollten sich für Frieden und Wohlfahrtssteigerung in einer neuen diskriminierungsverbietenden Gemeinschaft der Demokratien zusammenfinden, die als Lehre aus der glücklosen Friedensordnung des

5 Seit dem Inkrafttreten des Vertrags über die Europäische Union (Vertrag von Maastricht) am 1. 11. 1993 heißt die EWG schlicht EG.

6 Kilian, Europäisches Wirtschaftsrecht, München 1996, S. VII (ist in der 2. Aufl., 2003 nicht mehr enthalten).

7 Vgl. allein Art. 24 GG.

8 Die EGKS verfügte 1952-1957 über einen eigenen Gerichtshof als Vorläufer des EuGH. Die „Funktionalisten“ setzten sich dabei insgesamt gegen die „Föderalisten“ durch; s. Schulze, Anfänge und Ausbau der europäischen Integration - zu den Konzepten der frühen fünfziger Jahre, in: Hrbek/Schwarz (Hrsg.), 40 Jahre Römische Verträge: Der deutsche Beitrag, Berlin 1998, S. $91 \mathrm{ff}$.

9 Vgl. Taschner, Privatrechtsentwicklung durch die Europäische Gemeinschaft Rechtsgrundlagen, Ziele, Sachgebiete, Verfahren, in: Müller-Graff (Hrsg.), Gemeinsames Privatrecht in der Europäischen Gemeinschaft, 2. Aufl., Baden-Baden 1999, S. 225 (234); Joerges/Brüggemeier, Europäisierung des Vertragsrechts und Haftungsrechts, in: MüllerGraff, aaO, S. 301 (350 f.).

${ }^{10}$ Vgl. Behrens, Die Konvergenz der wirtschaftlichen Freiheiten im europäischen Gemeinschaftsrecht, EuR 1992, 145 (147); Reich, Bürgerrechte in der Europäischen Union, Baden-Baden 1999, S. 450 f. 
Versailler Vertrages zum Vorteil aller wirken sollte. Die Gründungsstaaten schufen mit dem am 25.3.1957 auf dem römischen Kapitol ${ }^{11}$ unterzeichneten Gründungsvertrag der Europäischen Wirtschaftgemeinschaft „die Grundlagen für einen immer engeren Zusammenschluß der europäischen Völker". ${ }^{12}$ Ausdruck erhält dieser Kooperationsgedanke bereits im sog. Schuman-Plan vom 9. 5. 1950. Es ging um die Erreichung konkreter und schrittweiser politischer Erfolge durch Zusammenarbeit, die einen allseitigen Anreiz zur Friedenserhaltung schaffen sollten. ${ }^{13}$ In dem SchumanPlan hieß es aber auch: „Die Zusammenlegung der Kohle- und Stahlproduktion wird unmittelbar die Grundlage gemeinsamer wirtschaftlicher Entwicklung schaffen als erste Etappe der europäischen Föderation“.

Ähnliches hebt auch Walter Hallstein (1901-1982) hervor, der zur EG als Schöpfung des Rechts schreibt: „Das ist das entscheidend Neue, was sie gegenüber früheren Versuchen auszeichnet, Europa zu einigen. Nicht Gewalt, nicht Unterwerfung ist als Mittel eingesetzt, sondern eine geistige, eine kulturelle Kraft, das Recht. Die Majestät des Rechts soll schaffen, was Blut und Eisen in Jahrhunderten nicht vermochten. Denn nur die selbstgewollte Einheit hat Aussicht auf Bestand, und Rechtsgleichheit und einheit sind untrennbar miteinander verbunden. Keine Rechtsordnung ohne Gleichheit vor dem Gesetz, Gleichheit aber bedeutet Einheit. Auf dieser Einsicht beruht der Vertrag von Rom, und darum schafft er eine Friedensordnung par excellence". .14

\section{Wandelungen im Europaverständnis}

Die erklärten Europäer der ersten Stunde waren - wie angedeutet - im Sinne einer negativen Referenz bewegt vom miterlittenen Krieg. Sie sahen vor fünfzig Jahren in der Schaffung eines Frieden und Wohlstand bringenden Europas auf dem wohl gewalterfahrendsten Erdteil der Welt eine Angelegenheit von Krieg oder Frieden. Mittlerweile ist jedoch ein Wandel eingetreten: War die erste Generation von Europapolitikern vom Kriegs- und Faschismuserlebnis geprägt (und teilweise von der Vision eines

${ }^{11}$ Im Saal der Horatier und Curatier des „Palazzo dei Conservatori“ auf dem Kapitolinischen Hügel, also dem Mittelpunkt des alten Roms.

${ }^{12}$ Eine ähnliche Formulierung wie in der Präambel des EWG- bzw. EG-Vertrages, aus der soeben zitiert wurde, und die Betonung des Plurals bei den „Völkern“ findet sich neuerdings auch in der Präambel der Grundrechtecharter (ABIEG Nr. C 364 v. 18. 12. 2000, S. 1 ff.): „Die Völker Europas sind entschlossen, auf der Grundlage gemeinsamer Werte eine friedliche Zukunft zu teilen, indem sie sich zu einer immer engeren Union verbinden".

${ }^{13} \mathrm{~S}$. auch Rösler, Ökonomische und politische Integrationskonzeptionen im Wettstreit - Zum Scheitern der Europäischen Verteidigungsgemeinschaft vor fünfzig Jahren, EuR 2005, 370 (371); weiter Schulze/Hoeren (Hrsg.), Dokumente zum Europäischen Recht, Bd. I: Gründungsverträge, Berlin 1999; Bd. 2: Justiz (bis 1957) Berlin 2000.

${ }^{14}$ Hallstein, Der unvollendete Bundesstaat, 4. Aufl., Düsseldorf u.a. 1969, S. 33. 
europäischen Staates beseelt), so ist die neue Generation weitenteils überzeugt, gemeinsame neue Probleme (etwa durch die Digitalisierung) seien eben nur durch gemeinsame Bemühungen zu lösen.

Dieses Verständnis der EU als ein Zweckverband herrscht heute auch in der Bevölkerung vor. Insofern ist der Machtverlust einzelner Staaten akzeptiert, um für den ,alten Kontinent" in einer gewandelten Staatenwelt eine neue wirtschaftliche und politische Bedeutung zu suchen. Derzeit wird die EU zumeist als Staatenverbund eingeordnet, so wie es auch das BVerfG in seiner grundlegenden Maastricht-Entscheidung von 1993 hervorhebt. ${ }^{15}$ Dennoch steht das reale (also nicht nur gewünschte) Endstadium noch offen (Bundesstaat oder Staatenbund? Vermischung der beiden, durch ein innovatives, nur in seiner spezifischen Qualität zu verstehendes Gebilde dritter Art, wie sich jetzt andeutet?).

\section{Andere Entgrenzungsbemühungen als solche durch die EU}

\section{Weitere Institutionen}

Auch außerhalb der EU-Strukturen und ihrer Vorläufer wurden auf dem europäischen Kontinent zahllose zwischen- und überstaatliche Organisationen geschaffen. Das älteste aktuelle europäische Regionalsystem ist der am 5. 5. 1949 gegründete Straßburger Europarat mit seinen derzeit 46 Signatarstaaten. Er hat die Konvention zum Schutze der Menschenrechte und Grundfreiheiten (EMRK vom 4. 11. 1950) ausgearbeitet, welche durch den 1959 eingerichteten Europäischen Gerichtshof für Menschenrechte (EGMR) behütetet wird. ${ }^{16}$ Die (wie beim EuGH) steigende Zahl ${ }^{17}$ der eingelegten Beschwerden, insbesondere aus den Staaten Zentral- und Osteuropas, beweist den Erfolg der Konvention. Zugleich bezeugt dies das gesamte Anliegen, auch auf internationaler Ebene die Gerichtsbarkeit zu Lasten und zur Kontrolle der Politik zu stärken, und zwar mittels (und mit dem Ergebnis) veröffentlichter regelorientierter und verfahrensmäßiger Grundsätze.

\footnotetext{
${ }^{15}$ BVerfGE 89, 155 (184 f., 188 ff.) - Maastricht: „Der EU-Vertrag begründet einen europäischen Staatenverbund, der von den Mitgliedstaaten getragen wird und deren nationale Identität achtet; er betrifft die Mitgliedschaft Deutschlands in supranationalen Organisationen, nicht eine Zugehörigkeit zu einem europäischen Staat"; näher Kirchhof, Die rechtliche Struktur der Europäischen Union als Staatenverbund, in: v. Bogdandy (Hrsg.), Europäisches Verfassungsrecht, Berlin u.a. 2003, S. 893 ff.; Barents, The Autonomy of Community Law, The Hague u.a. 2004; s. zuletzt im Jahre 2005 BVerfGE 113, 273 - Nichtigkeit des Europäischen Haftbefehlsgesetzes; dazu van Ooyen, Die Staatstheorie des Bundesverfassungsgerichts und Europa - Von Solange über Maastricht zum EU Haftbefehl, Baden-Baden 2006.

${ }^{16}$ Ausf. K. Ipsen, Individualschutz im Völkerrecht, in: ders. (Hrsg.), Völkerrecht, 5. Aufl., München 2004, Rn. 797 ff.

${ }^{17}$ Dazu Rösler, Zur Zukunft des Gerichtssystems der EU - Entwicklungstendenzen des EuGH zum Supreme Court Europas, ZRP 2000, 52 ff.
} 
Weiter zu erwähnen sind die 1961 gegründete Organisation für wirtschaftliche Zusammenarbeit und Entwicklung (Organization for Economic Co-operation and Development, kurz: OECD) ${ }^{18}$ die Organisation für Sicherheit und Zusammenarbeit in Europa (OSZE), die 1994 ins Leben gerufene WTO $^{19}$ als Nachfolger des 1947 gegründeten GATT ${ }^{20}$ und die durch die Unionserweiterungen recht bedeutungslos gewordene Europäische Freihandelszone (EFTA) und mit ihr der Europäische Wirtschaftsraum (EWR) als Zusammenarbeit von EG und EFTA. ${ }^{21}$

Dieses Geflecht an multilateralen Organisationen hat auch $\mathrm{zu}$ entscheidenden Veränderungen der klassischen Diplomatie geführt. Bilaterale diplomatische Beziehungen verlieren zusehends an Bedeutung, denn sowohl inner- als auch außerhalb der EU fallen unzählbare Entscheidungen auf internationalen Konferenzen, welche direkt von den nationalen Regierungen - unter verminderter Beteiligung der Botschaften vorbereitet und im Vorfeld abgestimmt werden. ${ }^{22}$ Des Weiteren bemühen sich die genannten Organisationen um so vielfältige Aufgaben wie Frieden und Abrüstung, ökonomischen Fortschritt, um die Herrschaft des Rechts und die Achtung der Menschenrechte und Grundfreiheiten.

Die Idee eines Zusammenschlusses von Staaten zu einer marktliberalen Kooperation geht über Europa hinaus, wie es sich etwa bei Nafta sowie Mercosor zeigt. Auch die neue panafrikanische Staatengemeinschaft (AU) orientiert sich ausdrücklich am Vorbild der EU. Es handelt sich also mit der staatlichen Kooperation um eine institutionelle Ausformung des global festzustellenden Siegeszugs des Rechts und eine Reaktion auf die Universalisierung der Märkte und die daraus resultierenden Einschränkungen der staatlichen Handlungsspielräume. ${ }^{23}$ Trotz ähnlicher Ansätze, für welche die EU Modell ist, ${ }^{24}$ bleibt die europäische

${ }^{18}$ Vormals OEEC; 1948 gegründet.

${ }^{19} 149$ Staaten sind mittlerweile Mitglied der WTO. Die VR China ist am 11.12. 2001 beigetreten.

${ }^{20}$ Allgemeines Zoll- und Handelsabkommen.

${ }^{21}$ Den EWR-Staaten sind Konsultativ-Beratungen z.B. im Vorfeld des Erlasses von EGVerbraucherrichtlinien eröffnet.

${ }^{22}$ Zum Wandel der deutschen Diplomatie s. Rösler, Die Botschaftsdiplomatie: Ein Einblick, Jura 1998, 220.

${ }^{23}$ Zur Antriebskraft globaler Märkte für internationale Beziehungen Schirm, Globale Märkte, nationale Politik und regionale Kooperation in Europa und den Amerikas, 2. Aufl., München 2001.

${ }^{24}$ Vgl. Schmitz, Integration in der supranationalen Union - Das europäische Organisationsmodell einer prozeßhaften geo-regionalen Integration und seine rechtlichen und staatstheoretischen Implikationen, Baden-Baden 2001, der die EU nicht als einmalig begreift, sondern sie als den ersten Vertreter einer staatsähnlichen Organisationsform des Überganges im Dreieck zwischen supranationaler Organisation, Staatenbund und Bundesstaat auffasst. S. weiter Pavón Piscitello/J. P. Schmidt, Der EuGH als Vorbild: Erste Entscheidung des ständigen Mercosur-Gerichts, EuZW 2006, 301 ff.; Vervaele, Mercosur 
Unternehmung wegen ihrer kulturhistorischen Vorprägung sowohl in Anspruch als auch vorzeigbaren Erfolgen dennoch gänzlich singulär.

\section{Außergemeinschaftlich initiiertes Privatrecht}

\section{a) Überblick zum wirtschaftsrelevanten Privatrecht}

$\mathrm{Zu}$ beachten sind die außerhalb des Unions- oder Gemeinschaftsrahmens eingegangenen Verpflichtungen durch völkerrechtliche Konventionen, ${ }^{25}$ die keinesfalls ein neues Phänomen darstellen. ${ }^{26}$ Die Revolutionierung des Verkehrs durch Einsatz von Dampfschiffen und Eisenbahnen hat im 19. Jahrhundert zu neuen Verflechtungen und zu ersten Ansätzen eines Weltmarktes geführt. Reaktion der Wirtschaftsstaaten hierauf war der Abschluss von Konventionen, so etwa der Pariser Übereinkunft zum Schutze des gewerblichen Eigentums (PVÜ) vom 20.3. $1883^{27}$ und die Berner Übereinkunft über den Eisenbahnfrachtverkehr vom 14. 10.1890. ${ }^{28}$ In dieser vom Imperialismus geprägten Zeit sind die Anfänge des Internationalismus zu finden, welcher mithin nicht erst mit der Gründung des Völkerbundes (1919) oder den Vereinten Nationen (1945) einsetzt. ${ }^{29}$

Eine internationale Gruppe um Michael Joachim Bonell vom 1926 auf italienische Initiative ins Leben gerufenen UNIDROIT ${ }^{30}$ in Rom hat 1994

and Regional Integration in South America, Int. Comp. Law. Q. 54 (2005), 387 ff.; Olivares Tramón, Das Vorabentscheidungsverfahren des EuGH als Vorbild des MERCOSUR, Baden-Baden 2006.

${ }^{25}$ Hierzu m. w. Beisp. Müller-Graff, Gemeinsames Privatrecht in der Europäischen Gemeinschaft - Ansatzpunkte, Ausgangsfragen, Ausfaltungen, in: ders. (Fn. 9), S. 9 (24 ff.); Basedow, Das BGB im künftigen europäischen Privatrecht: Der hybride Kodex Systemsuche zwischen nationaler Kodifikation und Rechtsvergleichung, AcP 200 (2000), 445 (454 f.).

${ }^{26}$ Solche Vereinbarungen sollten insoweit ebenfalls zum Europäischen Privatrecht gezählt werden; vgl. Basedow (Fn. 25), AcP 200 (2000), 445 (456, 457).

${ }^{27}$ RGB1. 1903, $147 \mathrm{ff}$.

${ }^{28}$ RGB1. 1892, 793 ff.; daneben bestehen etwa das Warschauer Übereinkommen von 1929 über den internationalen Luftverkehr, das Berner Übereinkommen von 1961 über den Eisenbahnfrachtverkehr, das Athener Übereinkommen von 1974 über den Seeverkehr und das Pariser Übereinkommen von 1962 über die Haftung der Gastwirte; zur Rechtsgleichheit auf Grund völkerrechtlicher Abkommen Kropholler, Internationales Einheitsrecht, Tübingen 1975.

${ }^{29}$ Vgl. Herren, Hintertüren zur Macht - Internationalismus und modernisierungsorientierte Außenpolitik in Belgien, der Schweiz und den USA 1865 - 1914, München 2000.

${ }^{30}$ Institut International pour l'Unification du Droit Privé; hierzu Rabel, Zwei Rechtsinstitute für die internationalen privatrechtlichen Beziehungen, JW 1932, 2225 (2227 f.); mit dem anderen Institut meint er das Kaiser-Wilhelm-Institut für ausländisches und internationales Privatrecht (Vorläufer des Max-Planck-Instituts, heute in Hamburg); zu den Ursachen der Gründung des Kaiser-Wilhelm-Instituts Basedow, Der Standort des Max-Planck-Instituts, in: Aufbruch nach Europa, Festschrift 75 Jahre Max-Planck-Institut für Privatrecht, Tübingen 2001, S. 3 ff.; Rösler, Siebzig Jahre Recht des Warenkaufs von Ernst Rabel - 
die „Principles of International Commercial Contracts“631 vorgelegt. Das in seiner Schlussfassung von 2004185 Artikel zählende Werk umfasst allgemeine Aspekte des Vertragsrechts für den internationalen Wirtschaftsverkehr. ${ }^{32}$ Diese Grundsätze für internationale Wirtschaftsverträge ähneln sich sowohl im Ansatz als auch in vielem Inhaltlichen ${ }^{33}$ mit den Grundsätzen des Europäischen Vertragsrechts der ehedem von Ole Lando geleiteten Kommission. ${ }^{34}$

Im wirtschaftsrelevanten Privatrecht spielen zusehends auch aufgezeichnete Gebräuche wie die 1953 von der Pariser ICC ${ }^{35}$ veröffentlichten transportrechtlichen Incoterms (International Commercial Terms), die Regeln über Dokumentenakkreditive, Definitionen von Klauseln

Werk- und Wirkgeschichte, RabelsZ 70 (2006), 793 ff.

${ }^{31}$ UNIDROIT Principles of International Commercial Contracts (2004), Rom 2004; deutsche Fassung in ZEuP 1997, 890 ff.; ZEuP 2005, 470 ff.; Schulze/Zimmermann (Hrsg.), Europäisches Privatrecht - Basistexte, 3. Aufl., Baden-Baden 2005, unter Abschn. III. 15.

${ }^{32}$ Bonell, UNIDROIT Principles 2004, in: Faust/Thüsing (Hrsg.), Beyond Borders: Perspectives on International and Comparative Law, Köln 2006, S. 5 ff.; ders., The UNIDROIT Principles in Practice, Ardsley 2006; Hartkamp, Principles of Contract Law, in: Hartkamp/Hesselink u.a. (Hrsg.), Towards a European Civil Code, 3. Aufl., Nijmegen 2004, S. 141 ff.; Brödermann, Die erweiterten UNIDROIT Prinzipien 2004, RIW 2004 721; Frick, Die UNIDROIT-Prinzipien für internationale Handelsverträge - ein noch unterschätzter Beitrag zur sog. Denationalisierung des Privatrechts, RIW 2001, 416 ff.; Basedow, Uniform Law Conventions and the UNIDROIT Principles of International Commercial Contracts, Uniform Law Review 2000, 129 (138) sieht in den Prinzipien zutreffend eine Reaktion auf die Krise des Einheitsprivatrechts.

${ }^{33} \mathrm{~S}$. für einen Vergleich Zimmermann, Die Unidroit-Grundregeln der internationalen Handelsverträge 2004 in vergleichender Perspektive, ZEuP 2005, 264 ff.; s. auch Canaris, Die Stellung der „UNIDROIT Principles“ und der „Principles of European Contract Law“ im System der Rechtsquellen, in: Basedow (Hrsg.), Europäische Vertragsrechtsvereinheitlichung und das deutsche Recht, Tübingen 2000, S. 5 ff.; Bonell, The UNIDROIT Principles of International Commercial Contracts and the Principles of European Contract Law: Similar Rules for the Same Purposes?, Uniform Law Review 1996, 229 ff.; zudem gehörte Lando eigentümlicher Weise auch der von Bonell geleiteten Kommission an; für eine tabellarische Gegenüberstellung Bonell/Peleggi, UNIDROIT Principles of International Commercial Contracts and Principles of European Contract Law: a Synoptical Table, Uniform Law Review 2004, 315.

${ }^{34}$ Lando/Beale (Hrsg.), Principles of European Contract Law, Part I and II, The Hague 2000; Lando/Clive/Prüm/Zimmermann (Hrsg.), Principles of European Contract Law, Part III, The Hague 2003; deutsche Fassung ZEuP 2000, 675 ff.; ZEuP 2003, 895 ff.; Schulze/Zimmermann (Fn. 31), Abschn. III. 10 und III. 11; dazu Zimmermann, Die Principles of European Contract Law als Ausdruck und Gegenstand europäischer Rechtswissenschaft, Jura 2005, 289 ff. und 441 ff.; weiter ders., Konturen eines europäischen Vertragsrechts, JZ 1995, 477 ff.; Lando, Das neue Schuldrecht des Bürgerlichen Gesetzbuches und die Grundregeln des Europäischen Vertragsrechts, RabelsZ 67 (2003) 231 ff.; Rösler, Der Griff nach dem Ungreifbaren - Zum Entstehen einer Europäischen Zivilrechtswissenschaft, KritV 2002, 392 (404 ff.).

${ }^{35}$ Die International Chamber of Commerce wurde 1920 als ,a businessmen's League of Nations" gegründet. 
und Termini, Musterverträge und sonstige einheitliche Richtlinien eine Rolle. ${ }^{36}$ Weiter bilden sich - etwa am Rhein - auf Grund ökonomischer Interessen grenzüberschreitende Regionen heraus, die nicht auf der Förderung oder Regulierung der Brüsseler Zentralgewalt beruhen. Auch auf Grund dieser Aspekte und der sich verbessernden Verkehrswirtschaft und Medientechnologie wird der Trend anhalten. Entsprechend hat sich auch eine eifrige Diskussion entwickelt, ob auf der Grundlage der genannten Regelungen sich insgesamt eine lex mercatoria, einschließlich der internationalen Handelsgerichtsbarkeit, herausbilden könnte, oder ob dies nicht schon längst geschehen ist. ${ }^{37}$

\section{b) UN-Kaufrecht}

Wichtigster Teil des Konventionsprivatrechts ist das Wiener Kaufrechtsabkommen (genau: Übereinkommen der Vereinten Nationen über Verträge über den internationalen Warenkauf vom 11. 4. 1980, englisch: UN Convention of the International Sale of Goods, abgekürzt: CISG). ${ }^{38}$ Das Übereinkommen gilt mittlerweile weltweit - teils mit erklärten Vorbehalten bezüglich bestimmter Artikel - in 69 Staaten. Es hat sich damit als eigenes, freilich noch partielles Sachrecht des internationalen Handelswarenkaufs, das in allen Vertragsstaaten unmittelbar auf gleiche Weise gelten soll (sog. Internationales Einheitsprivatrecht), in einem beachtlichen Maße durchgesetzt.

Gleichwohl gilt das CISG nicht einmal in allen EG-Staaten, denn das Vereinigte Königreich, Portugal und Irland sind nicht beigetreten. ${ }^{39}$ Beide

\footnotetext{
${ }^{36}$ Vgl. Berger, Einheitliche Rechtsstrukturen durch außergesetzliche Rechtsvereinheitlichung, JZ 1999, 369 ff.; Mertens, Nichtlegislatorische Rechtsvereinheitlichung durch transnationales Wirtschaftsrecht und Rechtsbegriff, RabelsZ 56 (1992), 219 ff.; zur „Autoharmonisation“ und der Aufgabe der Rechtsvergleichung Mansel, Rechtsvergleichung und europäische Rechtseinheit, JZ 1991, 529 (533f.).

${ }^{37}$ Zum Streitstand s. den Überblick (mit bejahendem Ergebnis) bei Lando, Die Regeln des Europäischen Vertragsrechts, in: Müller-Graff (Fn. 9), S. 567 (575 ff.); U. Stein, Lex mercatoria - Realität und Theorie, Frankfurt/M. 1995; Blaurock, Übernationales Recht des internationalen Handels, ZEuP 1993, 247 ff.; Basedow, Lex Mercatoria und Internationales Schuldvertragsrecht: eine rechtsökonomische Skizze, in: Festschrift Horn, Berlin 2006, S. 229 ff.; Lieckweg, Das Recht der Weltgesellschaft - systemtheoretische Perspektiven auf die Globalisierung des Rechts am Beispiel der Lex Mercatoria, Stuttgart 2003.

${ }^{38}$ BGB1 1989 II, 588, 1990 II, 1699; BGB1 1990 II, 1477, von der Bundesrepublik Deutschland am 26. 5. 1981 gezeichnet, am 21. 12. 1989 ratifiziert und dort am 1.1.1991 in Kraft getreten. Dem CISG ist mehr Erfolg gegönnt, da es - im Unterschied zum Haager Abkommen v. 1. 7. 1964 - allein den Handelsbereich betrifft (Art. 2) und zwischenzeitlich eine Gewöhnung an das in England ohnehin bekannte einheitliche Leistungsstörungsrecht eingetreten ist. S. zu den Entwicklungshintergründen Magnus, Wiener UN-Kaufrecht (CISG), in: Staudinger, Kommentar zum BGB, Neubearbeitung 2005, Berlin 2005; Schlechtriem/Schwenzer (Hrsg.), Kommentar zum Einheitlichen UN-Kaufrecht, 4. Aufl., München 2004; Rösler (Fn. 30), RabelsZ 70 (2006), 793 ff.

${ }^{39} \mathrm{~S}$. zu den Grenzen des CISG und sein Verhältnis zum Projekt eines Europäischen
} 
Parteien des Kaufvertrages über bewegliche Sachen müssen ihre Niederlassung in verschiedenen Kontraktsstaaten haben (Art. 1 Abs. 1) und sie dürfen das UN-Kaufrecht nicht wirksam ausgeschlossen haben (Art. 6). ${ }^{40}$ Ferner lässt das CISG weite Teile des nationalen Privatrechts unberührt, namentlich den Eigentumsübergang sowie die Gültigkeit des Vertrages, einschließlich der Wirksamkeitsfrage einer AGB-Klausel, die weiterhin durch das IPR - einem nationalen Recht zugewiesen wird (Art. 4).

Entscheidend ist weiter: Ausgeklammert sind mit Rücksicht auf nationale Verbraucherschutzvorschriften auch die Kaufverträge über Sachen, die lediglich für den persönlichen Gebrauch in Familie und Haushalt gedacht sind, also die Verbraucherkäufe (Art. 2 a). Während das Haager Abkommen für sämtliche bürgerlichrechtlichen Kaufverträge galt, verzichtet damit das CISG gänzlich auf die Einbeziehung von Verbraucherverträgen. Zugleich regelt das lückenhafte Übereinkommen weder die Gültigkeit des Vertrages oder einzelner Vertragsbestimmungen noch die Wirkungen, welche der Vertrag auf das Eigentum an der verkauften Ware hat (Art. 4). Das CISG findet ferner keine Anwendung auf die außervertragliche Haftung des Verkäufers (Art. 5).

Selbstverständlich wird das UN-Kaufrecht nicht nur auf unmittelbarem Weg im grenzüberschreitenden Verkehr berücksichtigt, sondern - da es anders als das EG-Recht gerade nicht für Inlandssachverhalte gilt - auch auf indirekte Weise, indem es zu einem interpretativen Zusammenwirken führt und bei Modifikationen des allgemeinen, rein für innerstaatliche Transaktionen geltenden nationalen Rechts (etwa bei der Schuldrechtsmodernisierung in Deutschland) eine Vorbildfunktion ausübt. ${ }^{41}$ Auch insofern ist die Idee eines rein nationalen Privatrechts gänzlich zur Illusion geworden. ${ }^{42}$ Allerdings fällt auf, wie sehr die hier beschriebenen Rechtsvereinheitlichungen für den kommerziellen Bereich geschaffen sind und daher - im Gegensatz zur EG - soziale Schutzpolitik und formalinstitutionelle Aspekte zur optimalen Durchsetzung des Rechts weitgehend außer Betracht lassen.

Zivilgesetzbuchs Bonell, The Need and Possibilities of a Codified European Contract Law, ERPL 1998, 505 (506 ff.).

${ }^{40}$ Dabei reicht nach deutscher Rechtsprechung die Vereinbarung des deutschen Vertragsrechts nicht aus; erforderlich ist vielmehr - da auch das CISG Teil der deutschen Rechtsordnung ist - eine Rechtswahl nach Art. 27 EGBGB gerade hinsichtlich des unvereinheitlichten deutschen Rechts.

${ }^{41}$ Über die Wirkung des UN-Kaufrechts Basedow, Zur weltweiten Konvergenz des Leistungsstörungsrechts - Der Einfluss des CISG, in: Festschrift für Georgiades, München 2005, S. 801 ff.; Mansel, Kaufrechtsreform in Europa und die Dogmatik des deutschen Leistungsstörungsrechts, AcP (204) 2004, 396 ff.

${ }^{42}$ Dies zeigt sich auch beim Entstehen einer transnationalen europäischen Zivilrechtswissenschaft mit inter- und intradisziplinären Bezügen, dazu Rösler (Fn. 34), KritV 2002, 392 (400 ff.). 


\section{Ursachen}

Somit ist die Illusionserosion eines an souveräner Autarkie orientierten Einzelstaates unbestreitbar. ${ }^{43}$ In der Tat sind die Nationalstaaten geschichtlich nicht ohne große Verdienste, schließlich ließen sich das Gewaltmonopol und der hierdurch ermöglichte Rechtsstaat sowie die Demokratie, die allgemeine Schulausbildung und das Gesundheitswesen allein im Nationalstaat - der auf enge Zusammengehörigkeit und daraus folgende Solidarität angelegt ist - dauerhaft realisieren. Nationalstaaten und nationale Identität werden daher ihre Existenzberechtigung und Identifikationsrolle für ihre Bürger auch in absehbarer Zukunft nicht verlieren. Jedoch relativiert sich mit der Lösung der Wirtschaft, Politik und Bürger aus den ortsgebundenen Kontextgegebenheiten der eigene Nationalstaat als Bezugspunkt. Die Klammern „Nation und Volk“ verlieren in verschiedenen gesellschaftlichen Teilbereichen wie Ökonomie, Politik, Wissenschaft, Kunst, Öffentlichkeit, Konsum und Populärkultur an Halt.

Zugleich wird der sich öffnende Einzelstaat diszipliniert und zusehends in ein Netz von regionenübergreifenden Neuschöpfungen und verbindenden universellen Werten eingewoben. In einer nicht mehr bipolaren Weltordnung zeigt sich die Annäherung an transnationale Organisationen selbst bei den strikt dem Neutralismus verpflichteten Staaten. Bestes Beispiel ist die Schweiz, deren Eidgenossen am 3. 3. 2002 zu 54,6 \% für eine Aufnahme in die UNO votierten, so dass sich Helvetien seit dem 10.9. 2002 als 190. Mitglied in der UNO findet. (Mittlerweile sind es insgesamt 192 Staaten.) Nach dem Grundsatz des autonomen Nachvollzugs des Gemeinschaftsrechts sind des Weiteren zahlreiche schweizerische Gesetze den Richtlinien und Verordnungen der EU nachgebildet. ${ }^{44}$ Auch die potenziellen Mitgliedstaaten bemühen sich um eine (freiwillige) Angleichung ihrer Privatrechtsordnungen. ${ }^{45}$

Neben der Intention einer säkularen Domestizierung aggressiver staatlicher Gewalt liegen auch die weiteren Gründe für den Sog zur Bildung übergeordneter zwischenstaatlicher Organisationen auf der Hand: Sie finden sich in den Bedürfnissen einer interdependenten, strukturell gewandelten

${ }^{43}$ Vgl. bereits Alfred Weber, Die Krise des modernen Staatsgedankens in Europa, Stuttgart 1925.

${ }^{44} \mathrm{Zu}$ den methodischen Herausforderungen Amstutz, Evolutorische Rechtsmethodik im europäischen Privatrecht - Zur richtlinienkonformen Auslegung und ihren Folgen für den autonomen Nachvollzug des Gemeinschaftsprivatrechts in der Schweiz, in: Werro/Probst (Hrsg.), Das schweizerische Privatrecht im Lichte des europäischen Gemeinschaftsrechts, Bern 2004, S. 105 (130 ff.); s. weiter zum Gedanken der Evolution Joerges, Europeanization as Process: Thoughts on the Europeanization of Private Law, European Public Law 11 (2005), 63.

${ }^{45}$ Etwa die Türkei $Y$. M. Atamer, Die autonome Umsetzung der Verbrauchsgüterkaufrichtlinie 1999/44/EG in der Türkei - zugleich ein Beitrag zum Stand des Verbraucherschutzes in der Türkei, ZEuP 2005, 566 ff. 
Weltökonomie, in der sich hinsichtlich Daten, Personen und Warengütern zunehmend vernetzenden und - problematischerweise nur partiell ${ }^{46}-$ entstehenden Weltgesellschaft ${ }^{47}$ sowie einer ins Bewusstsein geratenen Kleinheit, Begrenztheit und Schutzwürdigkeit des Erdballs, seiner Bewohner und Ressourcen, was $\mathrm{zu}$ einem mediengetragenen weltgleichzeitigen Empfinden über die nationalen Grenzen beigetragen hat.

Ebenso wichtige Kehrseite ist das sich globusumspannend angleichende Konsumenteninteresse an ausländischen Produktions- und Kulturgütern sowie das Verlangen nach fremden, medienvermittelten Nachrichten. Insofern besteht ein wechselwirkungsmäßiger Zusammenhang zwischen der menschlichen Neugier, dem Wunsch nach einem höheren Lebensstandard (das heißt eben auch stärkerem und bedürfnisausdifferenziertem Konsum bei gleichwohl zunehmender Standardisierung und Markenorientierung der Verbraucherpräferenzen ${ }^{48}$ ), dem globalen Kapitalismus und dem mit dieser Konstellation einhergehenden Zerbröckeln einzelstaatlicher Souveränität. Eine wirtschaftspatriotische Vorstellung, es müsse die heimische Wirtschaft gefördert werden oder eigene Produkte seien ausländischen per se überlegen, spielt beim Verbraucher zusehends eine gänzlich untergeordnete Rolle. ${ }^{49}$ Gerade in der neutralen Rolle als Konsument unterwandert der Mensch faktisch den Nationalgedanken. Der Verbraucher ist damit ebenfalls zu einer der Wirkkräfte für den Zerfall des Nationalstaates geworden.

Die sowohl im ökonomischen als auch privaten Bereich auftretenden Internationalisierungs-, Immaterialisierungs- und Individualisierungstendenzen, gepaart mit globaler technischer, unternehmerischer und auch rechtlicher Standardisierung (z.B. bei der Computer- und Kommunikationstechnologie, ${ }^{50}$ der Containerschifffahrt ${ }^{51}$ und dem Flugverkehr ${ }^{52}$ ) haben $\mathrm{zu}$ gravierenden Denationalisierungen ${ }^{53}$ geführt.

${ }^{46}$ Nicht zuletzt auch wegen des sog. digitalen Grabens.

${ }^{47}$ S. Luhmann, Ausdifferenzierung des Rechts - Beiträge zur Rechtssoziologie und Rechtstheorie, Frankfurt/M. 1999, S. 90 f.; die Aufsatzsammlungen Stichweh, Die Weltgesellschaft - soziologische Analysen, Frankfurt/M. 2000; spezieller di Fabio, Der Verfassungsstaat in der Weltgesellschaft, Tübingen 2001.

${ }^{48}$ S. zur Globalisierung von Märkten Levitt, The Globalization of Markets, Harvard Business Review 1983, 92 ff.

${ }^{49}$ Insofern lässt sich durch Steigerung des Konsums allein kaum eine nachhaltige Verbesserung am Arbeitsmarkt erwarten; dazu M. Kröger/Rösler, Grenzen und Chancen des Rechts zur Steuerung des Arbeitsmarktes, ZRP 2001, 473 (475).

${ }^{50}$ Zum Problem der Entgrenzung im Internet anhand des „P2P file sharing“ Rösler, Haftung von Medientauschbörsen und ihrer Nutzer in Nordamerika, Australien und Europa, MMR 2006, 503 ff.; zu Problemen des Internethandels ders., Anti-Counterfeiting in Online Auctions from the Perspective of Consumers' Interests, IIC 2006, 771.

${ }^{51}$ Vor fünfzig Jahre, nämlich 1956, wurde zum ersten Mal ein Container eingesetzt; s. Levinson, The Box: How the Shipping Container Made the World Smaller and the World Economy Bigger, Princeton u.a. 2006.

${ }^{52}$ Dies wird auch durch das europäische Wirtschaftsrecht aktiv unterstützt; s. etwa Rösler, 
Sowohl die in räumlicher, sachlicher und persönlicher Hinsicht stattfindenden Vereinheitlichungen, ${ }^{54}$ als auch die neuen Unübersichtlichkeiten $^{55}$ auf Grund autonom agierender, sich aus einem staatlichen Rahmen herauslösender Unternehmen, Gruppierungen und Individuen, die damit keinen klar definierbaren Adressaten abgeben, sind eine ernste Herausforderung an die staatliche und überstaatliche Regelungsund Steuerungskapazität. ${ }^{56}$

Um diese Kapazitäten nicht zu verlieren, muss das Recht in der „postnationalen Konstellation“ (Jürgen Habermas) die bereits transnationalisierte Lebenswirklichkeit der Wirtschaft und zu geringem Umfang der Individuen gleichsam einholen. ${ }^{57}$ Die nationalen Institutionen in ihrer bisherigen Form sind auf Grund dieser Erkenntnis kritisch zu hinterfragen. Einhergehend wird angesichts global agierender Unternehmen und zwischenstaatlicher Organisationen ein Demokratiedefizit offenbar. Sie entziehen sich staatlicher „Bevormundung“ schlicht durch Sitzverlegung. Die EU wäre in der Lage, hier teilweise ausgleichend zu wirken, da sie - bei entsprechender Ausgestaltung als Mehrebenen-Demokratie - dem Bürger die reelle Chance eröffnen könnte, auch im erweiterten Rahmen kollektive Freiheit und Mitbestimmung auszuüben. ${ }^{58}$ Unzutreffend ist es daher am Maßstab des mächtigen Nationalstaates den Steuerungsverlust desselben zu

Billigfluglinien im EU-Wirtschaftsrecht - Marktordnungs-, Beihilfe- und Fluggastrecht, ZHR 170 (2006), $336 \mathrm{ff}$.

${ }^{53} \mathrm{~S}$. zur ihr speziell Remien, Denationalisierung des Privatrechts in der Europäischen Union? - Legislative oder gerichtliche Wege, ZfRV 1995, 116 f.; Kreuzer, Entnationalisierung des Privatrechts durch globale Rechtsintegration?, in Raum und Recht, Festschrift 600 Jahre Würzburger Juristenfakultät, Berlin 2002, S. 247; ihm zu Ehren Kronke, Herausforderungen internationaler Privatrechtsmodernisierung, in: Kieninger (Hrsg.), Denationalisierung des Privatrechts?, Tübingen 2005, S. 53; weiter Denninger, Vom Ende nationalstaatlicher Souveränität in Europa, JZ 2000, 1121 ff.; de Búrca/Gerstenberg, The Denationalization of Constitutional Law, Harvard International Law Journal 47 (2006), 243 ff.

${ }^{54}$ S. hierfür Rehbinder, Rechtssoziologie, 5. Aufl., München 2003, S. 102 ff.

${ }^{55}$ Entlehnt von Habermas, Die Neue Unübersichtlichkeit, Kleine politische Schriften V, Frankfurt/M. 1985; zum veränderten Umfeld in diesem Sinne Ritter, Das Recht als Steuerungsmedium im kooperativen Staat, in: Grimm (Hrsg.), Wachsende Staatsaufgaben sinkende Steuerungsfähigkeit des Rechts, Baden-Baden 1990, S. 69 (71 f.).

${ }^{56}$ Zur Privatisierungstendenz durch nichtstaatliche Organisationen (NGOs) und transnationale Unternehmen (TNCs) sowie zur dagegen vorangetriebenen Verregelung s. Brühl (Hrsg.), Die Privatisierung der Weltpolitik, Bonn 2001; speziell zur Frage der globalen Vereinheitlichung des Rechts der Wettbewerbsbeschränkungen Basedow, Weltkartellrecht, Tübingen 1998.

${ }^{57}$ Habermas, Die Postnationale Konstellation, Frankfurt/M. 1998, S. 84 f., 79, er hat damit vor allem die - unverzichtbare - Schaffung einer europäischen Demokratie im Sinn, s. dazu insbes. S. 91 ff.; s. weiter Michaels, Globale Wirtschaft und lokale Gesetzgebung, ZVglRWiss 2001, $182 \mathrm{ff}$.

${ }^{58}$ Vgl. auch Zürn, Regieren jenseits des Nationalstaates: Globalisierung und Denationalisierung als Chance, 2. Aufl., Frankfurt/M. 2005. 
beklagen, ohne sich die hierdurch eröffnenden Chancen kooperativer Modelle vor Augen zu führen.

\section{Gesamtbetrachtung}

Die vorbildgebende Europäische Gemeinschaft hat sich ganz im Sinne des von Immanuel Kant (1724-1804) in seiner Schrift „Zum ewigen Frieden“ geforderten „Föderalism freier Staaten“ als Grundlage des Völkerrechts bzw. eines „Völkerbund[es], der aber gleichwohl kein Völkerstaat sein müßte“, ${ }^{59}$ gebildet und entwickelt. Zugleich hat Kant dort die verbindende Kraft des Handels hervorgehoben. Zusehends finden sich Staaten weltweit - trotz mancher Rückschläge - in kooperativer, regelgebundener und helfender Weise zusammen und erkennen letztlich in der Öffnung ihrer Märkte, leichter als in anderen Bereichen, einen gemeinsamen Nenner.

Dieser vom Handelgeist getragene neue Staatstypus ist durch die Bereitschaft zum Autonomieverlust, durch Multilateralismus, die Präferenz ziviler Instrumente bei der staatlichen Interessenverfolgung sowie dem Primat der Wohlstandserhöhung gekennzeichnet. ${ }^{60}$ Er schließt - im Fall einer höheren Stufe der Staatenkooperation - auch sozial-privatrechtliche und demokratische Kofaktoren ein. Aufgedeckt wird für die Rechtswissenschaft zugleich, dass isolationistische Tendenzen unhaltbar $\operatorname{sind}^{61}$ und das Privatrecht nicht an Nationalstaat und Volksgeist ${ }^{62}$ gebunden sein muss. In diesem Bereich (sogar außerhalb der relativ festen Klammer der EU) bestehen unübersehbare allgemeine Entgrenzungstrends, welche ihrerseits auf ein Fortschreiten des Integrationsprozesses der Union hindeuten - den erfolgten und noch anstehenden Erweiterungen und der insofern innerhalb der EU zunehmenden Multiplikation von Interessen zum Trotz.

Aufgebrochen wird die Innenperspektive der Nation - dies auch ohne den Druck der vornehmlich exportorientierten Wirtschaftszweige: Als Gegenstücke der Internationalisierung des Wirtschafts- und Arbeitslebens

${ }^{59}$ Kant, Zum ewigen Frieden, Zweiter Abschnitt, Zweiter Definitivartikel zum ewigen Frieden, Königsberg/Berlin 1795/1995, S. 16; Slaughter, International Law in a World of Liberal States, European Journal of International Law (EJIL) 1995, 503 ff.; kritisch zu dieser modernen Rezeption von Kants Auffassung Alvarez, Do Liberal States Behave Better?, EJIL 2001, 183 ff.; vgl. auch Höffe, Vernunft und Recht - Bausteine zu einem interkulturellen Rechtsdiskurs, 2. Aufl., Frankfurt/M. 1998, wonach Zivilisationskonflikte der Völker als Ausdruck der sozialen Vernunft interkulturell durch Recht zu lösen sind.

${ }^{60}$ Vgl. Staack, Handelsstaat Deutschland - Außenpolitik in einem neuen internationalen System, Paderborn u.a. 2000.

${ }^{61} \mathrm{~S}$. zu den ausländischen Einflussfaktoren auf die Schaffung der Kodifikationen Rösler, Rechtsvergleichung als Erkenntnisinstrument in Wissenschaft, Praxis und Ausbildung (Teil 2), JuS 1999, 1186 (1187).

${ }^{62} \mathrm{Zu}$ den Unklarheiten des von $v$. Savigny (1779-1861) entwickelten Konzepts s. Rösler, (Teil 1), JuS 1999, 1084 (1085); Koschaker, Europa und das römische Recht, 4. Aufl., München u.a. $1966 / 1976$, S. $198 \mathrm{f}$. 
finden sich die Entgrenzung des Tourismus, der privaten Kommunikation, der Wissenschaft und Ausbildung. Betroffen ist die gesamte Werteebene, was sich auch an der Stärkung universalistischer Gerechtigkeitsprinzipien wie z.B. den Menschenrechten zeigt, die übergeordneten Geltungsanspruch erheben. Derart bilden sich supranationale Verhaltensstrukturen, -standards und damit -erwartungen heraus. Doch wie steht es um den Schutz derselben?

Vielfach kranken Grundsätze der lex mercatoria ebenso wie die Prinzipienwerke und weite Teile des internationalen Rechts an der Rechtsdurchsetzung. Zahlreiche Regelungen werden nur als Option empfohlen, ${ }^{63}$ was etwa in sensiblen Bereichen mit markt- und verhandlungsunterlegenen Parteien nicht angemessen scheint. Weiter mangelt es an einer einheitlichen Auslegung und einer letztverbindlichen Kontrollinstanz. Deutlich wird dies im UN-Kaufrecht z.B. bei der ergebnisrelevanten Frage, was die geschuldete Leistung i.S.d. Art. 35 Abs. 2 lit. a CISG sein soll: Während im englischen Common Law die handelbare Qualität als Maßstab bevorzugt wird, soll im germanischen Rechtskreis tendenziell die durchschnittliche Qualität maßgeblich sein, ${ }^{64}$ wohingegen sich kürzlich ein niederländisches Schiedsgericht für vernünftige (,reasonable“) Warenqualität zur Erfüllung des Konformitätserfordernisses ausgesprochen hat. ${ }^{65}$

Einmal mehr zeigt sich mit der obligatorischen Wirkung des Gemeinschaftsrechts und dem um autonome Auslegung bemühten $\mathrm{EuGH}^{66}$ die Stärke der „über“ den mitgliedgliedstaatlichen Rechtstraditionen gebauten $^{67}$ EU. Ihr vergleichsweise hoher Entwicklungsstand ${ }^{68}$ liegt darin

${ }^{63}$ Näher Schwartze, Europäische Sachmängelgewährleistung beim Warenkauf, Tübingen 2000, S. 601.

${ }^{64}$ Vgl. auch ablehnend BT-Drs. $14 / 6040$ v. 14.5.2001, S. 214 im Fall der Schuldrechtsmodernisierung des BGB: der dem deutschen Gesetzbuch „fremde“ Begriff „vernünftigerweise“ solle keinen Eingang finden; damit entschieden sich die deutschen Gesetzverfasser anders als der Gemeinschaftsgesetzgeber in Art. 2 II d) der Verbrauchsgüterrichtlinie (Richtlinie 1999/44/EG, ABl. EG Nr. L 171, S. 12 ff.).

${ }^{65}$ Netherlands Arbitration Institute (Rotterdam), Urt. v. 15. 10. 2002, Nr. 2319, Text-Nr. 61 ff. - Rijn Blend, m. w. Nachw.; verfügbar über http://www.unilex.info. Ferrari, Divergences in the application of the CISG's rules on non-conformity of goods, RabelsZ 68 (2004), $473 \mathrm{ff}$.

${ }^{66}$ Näher Basedow/Rösler, Wege zur Privatrechtseinheit in Europa, Jura 2006, 228 ff.; Rösler, Die Anwendung von Prinzipien des europäischen Verbraucherprivatrechts in der jüngeren EuGH-Rechtsprechung, ZEuS 2006, 341 ff.; zur Verantwortung der mitgliedstaatlichen Gerichte Basedow, Nationale Justiz und Europäisches Privatrecht, Heidelberg 2003, S. 6 ff.

${ }^{67}$ Dazu Rösler, Großbritannien im Spannungsfeld europäischer Rechtskulturen, ZVglRWiss 2001, 448 (458 ff.).

${ }^{68}$ Dies gerade bei der sozusagen sozial geprägten Materie des Verbraucherschutzes aufzeigend Rösler, Europäische Integration durch Verbraucherschutz: Entwicklungsursachen und Beschränkungen, VuR 2003, 12 ff.; ders., 30 Jahre Verbraucherpolitik in Europa - rechtsvergleichende, programmatische und institutionelle Faktoren, ZfRV 2005, 134 ff. 
begründet, dass sich die europäischen Bemühungen gerade nicht im Abbau von Komplexität (etwa zur Vereinfachung von grenzüberschreitenden Vertragsabschlüssen und der grenzüberschreitenden Rechtsverfolgung) erschöpfen, sondern, dass sie kulturhistorisch auf spezifische Art und Weise unterfüttert sind mit dem vermächtnishaften Sinngebungs- und Interpretationsmuster eines durch Recht fortschreitend integrativ $\mathrm{zu}$ befriedenden Europas. Dies gilt auch für die Erweiterung der EU um zehn ost- und mitteleuropäische Staaten am 1. 5. 2004, welche sich letztlich als Überwindung der in Jalta im Februar 1945 kriegs- und ideologiebedingt vereinbarten Teilung Europas darstellt.

$\mathrm{Zu}$ erwähnen bleibt als Ausblick auf die weitere Privatrechtsentwicklung in Europa: Auf Anregung der Europäischen Kommission in Brüssel wird derzeit ein sog. Gemeinsamer Referenzrahmen ausgearbeitet ${ }^{69}$. Er soll u.a. die zutreffend kritisierten Inkohärenzen $^{70}$ zwischen den verschiedenen Richtlinien des Privatrechts ausbessern ${ }^{71}$. Noch ist unklar, ob diese Ausarbeitungen, welche insbesondere auf die Entwürfe der LandoKommission zurückgreifen, die Vorläufer zu einem Europäischen Zivilgesetzbuch darstellen können. Allein die Furcht, dem könnte so sein, hat einige Skepsis gegenüber dem Projekt hervorgerufen; ${ }^{72}$ schließlich würde sich dies derzeit noch schwer mit den mittlerweile 25 verschiedenen Rechtskulturen in der EU vertragen, die notwendigerweise einen kooperativen und koevolutiven Charakter der Privatrechtsintegration erfordern.

\footnotetext{
${ }^{69}$ S. zuletzt die Mitteilung der Kommission an das europäische Parlament und den Rat: Europäisches Vertragsrecht und Überarbeitung des gemeinschaftlichen Besitzstands weiteres Vorgehen, KOM (2004) 651 endg. v. 11. 10. 2004; Bericht der Kommission: Erster jährlicher Fortschrittsbericht zum europäischen Vertragsrecht und zur Überprüfung des gemeinschaftlichen Besitzstands, KOM (2005) 456 endg. v. 23. 9. 2005. Weiter McGuire, Ziel und Methode der Study Group on a European Civil Code, ZfRV 2006, 163 174.

${ }^{70}$ Näher etwa Rösler (Fn. 3), S. 218 ff.

${ }^{71}$ Staudenmayer, Weitere Schritte im Europäischen Vertragsrecht, EuZW 2005, 103 ff.; Lando, Der Aktionsplan der EG-Kommission zum europäischen Vertragsrecht, RIW 2005, $1 \mathrm{ff}$.

${ }^{72}$ S. darüber hinaus zur Sorge, es würde ein zu wirtschaftsnahes, unsoziales Recht entstehen Study Group on Social Justice in European Private Law, Social Justice in European Contract Law: a Manifesto, European Law Journal 10 (2004), 653 ff.
} 


\section{Literaturvezeichnis}

ALVAREZ: Do Liberal States Behave Better?, EJIL 2001, 183.

AMSTUTZ: Evolutorische Rechtsmethodik im europäischen Privatrecht - Zur richtlinienkonformen Auslegung und ihren Folgen für den autonomen Nachvollzug des Gemeinschaftsprivatrechts in der Schweiz, in: WERRO/PROBST (Hrsg.), Das schweizerische Privatrecht im Lichte des europäischen Gemeinschaftsrechts, Bern 2004, S. 105.

ATAMER, Y. M.: Die autonome Umsetzung der Verbrauchsgüterkaufrichtlinie 1999/44/EG in der Türkei - zugleich ein Beitrag zum Stand des Verbraucherschutzes in der Türkei, ZEuP 2005, 566.

BARENTS: The Autonomy of Community Law, The Hague u.a. 2004.

BASEDOW: Das BGB im künftigen europäischen Privatrecht: Der hybride Kodex Systemsuche zwischen nationaler Kodifikation und Rechtsvergleichung, AcP 200 (2000), 445.

BASEDOW: Der Standort des Max-Planck-Instituts, in: Aufbruch nach Europa, Festschrift 75 Jahre Max-Planck-Institut für Privatrecht, Tübingen 2001, S. 3.

BASEDOW: Grundlagen des europäischen Privatrechts, JuS 2004, 89.

BASEDOW: Lex Mercatoria und Internationales Schuldvertragsrecht: eine rechtsökonomische Skizze, in: Festschrift Horn, Berlin 2006, S. 229.

BASEDOW: Nationale Justiz und Europäisches Privatrecht, Heidelberg 2003, S. 6.

BASEDOW: Uniform Law Conventions and the UNIDROIT Principles of International Commercial Contracts, Uniform Law Review 2000, 129.

BASEDOW: Weltkartellrecht, Tübingen 1998.

BASEDOW: Zur weltweiten Konvergenz des Leistungsstörungsrechts - Der Einfluss des CISG, in: Festschrift für Georgiades, München 2005, S. 801.

BASEDOW/KONO (Hrsg.): Legal Aspects of Globalization, The Hague u.a. 2000.

BASEDOW/RÖSLER: Wege zur Privatrechtseinheit in Europa, Jura 2006, 228.

BEHRENS: Die Konvergenz der wirtschaftlichen Freiheiten im europäischen Gemeinschaftsrecht, EuR 1992, 145.

BERGER: Einheitliche Rechtsstrukturen durch außergesetzliche Rechtsvereinheitlichung, JZ 1999, 369.

BLAUROCK: Übernationales Recht des internationalen Handels, ZEuP 1993, 247.

BONELL: The Need and Possibilities of a Codified European Contract Law, ERPL 1998, 505.

BONELL: The UNIDROIT Principles in Practice, Ardsley 2006.

BONELL: The UNIDROIT Principles of International Commercial Contracts and the Principles of European Contract Law: Similar Rules for the Same Purposes?, Uniform Law Review 1996, 229.

BONELL: UNIDROIT Principles 2004, in: FAUST/THÜSING (Hrsg.), Beyond Borders: Perspectives on International and Comparative Law, Köln 2006, S. 5. 
BONELL/PELEGGI: UNIDROIT Principles of International Commercial Contracts and Principles of European Contract Law: a Synoptical Table, Uniform Law Review 2004, 315.

BRÖDERMANN: Die erweiterten UNIDROIT Prinzipien 2004, RIW 2004, 721.

BRÜHL (Hrsg.): Die Privatisierung der Weltpolitik, Bonn 2001.

CANARIS: Die Stellung der „UNIDROIT Principles“ und der „Principles of European Contract Law“ im System der Rechtsquellen, in: BASEDOW (Hrsg.), Europäische Vertragsrechtsvereinheitlichung und das deutsche Recht, Tübingen 2000, S. 5.

DE BÚRCA/GERSTENBERG: The Denationalization of Constitutional Law, Harvard International Law Journal 47 (2006), 243.

DENNINGER: Vom Ende nationalstaatlicher Souveränität in Europa, JZ 2000, 1121.

DI FABIO: Der Verfassungsstaat in der Weltgesellschaft, Tübingen 2001.

FERRARI: Divergences in the application of the CISG's rules on non-conformity of goods, RabelsZ 68 (2004), 473.

FRICK: Die UNIDROIT-Prinzipien für internationale Handelsverträge - ein noch unterschätzter Beitrag zur sog. Denationalisierung des Privatrechts, RIW 2001, 416.

GRUNDMANN: Europäisches Schuldvertragsrecht - Struktur und Bestand, NJW 2000, 14.

HABERMAS: Die Neue Unübersichtlichkeit, Kleine politische Schriften V, Frankfurt/M. 1985.

HABERMAS: Die Postnationale Konstellation, Frankfurt/M. 1998.

HALLSTEIN: Der unvollendete Bundesstaat, 4. Aufl., Düsseldorf u.a. 1969, S. 33.

HARTKAMP: Principles of Contract Law, in: HARTKAMP/HESSELINK U.A. (Hrsg.), Towards a European Civil Code, 3. Aufl., Nijmegen 2004, S. 141.

HEIDERHOFF: Gemeinschaftsprivatrecht, München 2005.

HERREN: Hintertüren zur Macht - Internationalismus und modernisierungsorientierte Außenpolitik in Belgien, der Schweiz und den USA 1865 - 1914, München 2000.

HÖFFE: Vernunft und Recht - Bausteine zu einem interkulturellen Rechtsdiskurs, 2. Aufl., Frankfurt/M. 1998.

JOERGES: Europeanization as Process: Thoughts on the Europeanization of Private Law, European Public Law 11 (2005), 63.

JOERGES/BRÜGGEMEIER: Europäisierung des Vertragsrechts und Haftungsrechts, in: MÜLLER-GRAFF, MÜLLER-GRAFF (Hrsg.), Gemeinsames Privatrecht in der Europäischen Gemeinschaft, 2. Aufl., Baden-Baden 1999, S. 301.

IPSEN, K.: Individualschutz im Völkerrecht, in: DERS. (Hrsg.), Völkerrecht, 5. Aufl., München 2004, Rn. 797.

KANT: Zum ewigen Frieden, Königsberg/Berlin 1795/1995.

KILIAN: Europäisches Wirtschaftsrecht, München 1996. 
KIRCHHOF: Die rechtliche Struktur der Europäischen Union als Staatenverbund, in: V. BOGDANDY (Hrsg.), Europäisches Verfassungsrecht, Berlin u.a. 2003, S. 893.

KOSCHAKER: Europa und das römische Recht, 4. Aufl., München u.a. 1966/1976.

KREUZER: Entnationalisierung des Privatrechts durch globale Rechtsintegration?, in Raum und Recht, Festschrift 600 Jahre Würzburger Juristenfakultät, Berlin 2002, S. 247.

KRONKE: Herausforderungen internationaler Privatrechtsmodernisierung, in: KIENINGER (Hrsg.), Denationalisierung des Privatrechts?, Tübingen 2005, S. 53.

KROPHOLLER: Internationales Einheitsrecht, Tübingen 1975.

KRÖGER, M. /RÖSLER: Grenzen und Chancen des Rechts zur Steuerung des Arbeitsmarktes, ZRP 2001, 473 (475).

LANDO: Das neue Schuldrecht des Bürgerlichen Gesetzbuches und die Grundregeln des Europäischen Vertragsrechts, RabelsZ 67 (2003) 231.

LANDO: Der Aktionsplan der EG-Kommission zum europäischen Vertragsrecht, RIW 2005, 1.

LANDO: Die Regeln des Europäischen Vertragsrechts, in: MÜLLER-GRAFF (Hrsg.), Gemeinsames Privatrecht in der Europäischen Gemeinschaft, 2. Aufl., Baden-Baden 1999, S. 567.

LANDO/BEALE (Hrsg.): Principles of European Contract Law, Part I and II, The Hague 2000.

LANDO/CLIVE/PRÜM/ZIMMERMANN (Hrsg.): Principles of European Contract Law, Part III, The Hague 2003.

LEVINSON: The Box: How the Shipping Container Made the World Smaller and the World Economy Bigger, Princeton u.a. 2006.

LEVITT: The Globalization of Markets, Harvard Business Review 1983, 92.

LIECKWEG: Das Recht der Weltgesellschaft - systemtheoretische Perspektiven auf die Globalisierung des Rechts am Beispiel der Lex Mercatoria, Stuttgart 2003.

LUHMANN: Ausdifferenzierung des Rechts - Beiträge zur Rechtssoziologie und Rechtstheorie, Frankfurt/M. 1999, S. 90.

MANSEL: Kaufrechtsreform in Europa und die Dogmatik des deutschen Leistungsstörungsrechts, AcP (204) 2004, 396.

MANSEL: Rechtsvergleichung und europäische Rechtseinheit, JZ 1991, 529.

MCGUIRE: Ziel und Methode der Study Group on a European Civil Code, ZfRV 2006, 163.

MERTENS: Nichtlegislatorische Rechtsvereinheitlichung durch transnationales Wirtschaftsrecht und Rechtsbegriff, RabelsZ 56 (1992), 219.

MICHAELS: Globale Wirtschaft und lokale Gesetzgebung, ZVgIRWiss 2001, 182.

MÜLLER-GRAFF: Gemeinsames Privatrecht in der Europäischen Gemeinschaft Ansatzpunkte, Ausgangsfragen, Ausfaltungen, in: DERS. (Hrsg.), Gemeinsames Privatrecht in der Europäischen Gemeinschaft, 2. Aufl., Baden-Baden 1999, S. 9. 
OLIVARES TRAMÓN: Das Vorabentscheidungsverfahren des EuGH als Vorbild des MERCOSUR, Baden-Baden 2006.

PAVÓN PISCITELLO/SCHMIDT, J. P.: Der EuGH als Vorbild: Erste Entscheidung des ständigen Mercosur-Gerichts, EuZW 2006, 301.

RABEL: Zwei Rechtsinstitute für die internationalen privatrechtlichen Beziehungen, JW 1932, 2225.

REHBINDER: Rechtssoziologie, 5. Aufl., München 2003.

REICH: Bürgerrechte in der Europäischen Union, Baden-Baden 1999.

REICH/MICKLITZ: Europäisches Verbraucherrecht, 4. Aufl., Baden-Baden 2003.

REMIEN: Denationalisierung des Privatrechts in der Europäischen Union? Legislative oder gerichtliche Wege, ZfRV 1995, 116.

RITTER: Das Recht als Steuerungsmedium im kooperativen Staat, in: GRIMM (Hrsg.), Wachsende Staatsaufgaben - sinkende Steuerungsfähigkeit des Rechts, Baden-Baden 1990, S. 69.

RÖSLER: Anti-Counterfeiting in Online Auctions from the Perspective of Consumers' Interests, IIC 2006, 771.

RÖSLER: 30 Jahre Verbraucherpolitik in Europa - rechtsvergleichende, programmatische und institutionelle Faktoren, ZfRV 2005, 134.

RÖSLER: Billigfluglinien im EU-Wirtschaftsrecht - Marktordnungs-, Beihilfe- und Fluggastrecht, ZHR 170 (2006), 336.

RÖSLER: Der Griff nach dem Ungreifbaren - Zum Entstehen einer Europäischen Zivilrechtswissenschaft, KritV 2002, 392.

RÖSLER: Die Anwendung von Prinzipien des europäischen Verbraucherprivatrechts in der jüngeren EuGH-Rechtsprechung, ZEuS 2006, 341.

RÖSLER: Die Botschaftsdiplomatie: Ein Einblick, Jura 1998, 220.

RÖSLER: Europäische Integration durch Verbraucherschutz: Entwicklungsursachen und Beschränkungen, VuR 2003, 12.

RÖSLER: Europäisches Konsumentenvertragsrecht - Grundkonzeption, Prinzipien und Fortentwicklung, München 2004.

RÖSLER: Großbritannien im Spannungsfeld europäischer Rechtskulturen, ZVglRWiss 2001, 448.

RÖSLER: Haftung von Medientauschbörsen und ihrer Nutzer in Nordamerika, Australien und Europa, MMR 2006, 503.

RÖSLER: Ökonomische und politische Integrationskonzeptionen im Wettstreit Zum Scheitern der Europäischen Verteidigungsgemeinschaft vor fünfzig Jahren, EuR 2005, 370.

RÖSLER: Rechtsvergleichung als Erkenntnisinstrument in Wissenschaft, Praxis und Ausbildung (Teil 2), JuS 1999, 1186 (1187).

RÖSLER: Siebzig Jahre Recht des Warenkaufs von Ernst Rabel - Werk- und Wirkgeschichte, RabelsZ 70 (2006), 793.

RÖSLER: Zur Zukunft des Gerichtssystems der EU - Entwicklungstendenzen des EuGH zum Supreme Court Europas, ZRP 2000, 52.

SCHÄFER, W.: Globalisierung: Entmonopolisierung des Nationalen?, in: BERG, H. (Hrsg.), Globalisierung der Wirtschaft: Ursachen - Formen - 
Konsequenzen, (Schriften des Vereins für Socialpolitik, Neue Folge, Bd. 263), Berlin 1999, S. 9.

SCHIRM: Globale Märkte, nationale Politik und regionale Kooperation in Europa und den Amerikas, 2. Aufl., München 2001.

SCHLECHTRIEM/SCHWENZER (Hrsg.): Kommentar zum Einheitlichen UNKaufrecht, 4. Aufl., München 2004.

SCHMITZ: Integration in der supranationalen Union - Das europäische Organisationsmodell einer prozeßhaften geo-regionalen Integration und seine rechtlichen und staatstheoretischen Implikationen, Baden-Baden 2001.

SCHULZE: Anfänge und Ausbau der europäischen Integration - zu den Konzepten der frühen fünfziger Jahre, in: HRBEK/SCHWARZ (Hrsg.), 40 Jahre Römische Verträge: Der deutsche Beitrag, Berlin 1998, S. 91.

SCHULZE/HOEREN (Hrsg.): Dokumente zum Europäischen Recht, Bd. I: Gründungsverträge, Berlin 1999; Bd. 2: Justiz (bis 1957) Berlin 2000.

SCHULZE/ZIMMERMANN (Hrsg.): Europäisches Privatrecht - Basistexte, 3. Aufl., Baden-Baden 2005.

SCHWARTZE: Europäische Sachmängelgewährleistung beim Warenkauf, Tübingen 2000, S. 601.

SLAUGHTER: International Law in a World of Liberal States, EJIL 1995, 503.

STAACK: Handelsstaat Deutschland - Außenpolitik in einem neuen internationalen System, Paderborn u.a. 2000.

STAUDENMAYER: Weitere Schritte im Europäischen Vertragsrecht, EuZW 2005, 103.

STEIN, U.: Lex mercatoria - Realität und Theorie, Frankfurt/M. 1995.

STICHWEH: Die Weltgesellschaft - soziologische Analysen, Frankfurt/M. 2000.

STUDY GROUP ON SOCIAL JUSTICE IN EUROPEAN PRIVATE LAW: Social Justice in European Contract Law: a Manifesto, European Law Journal 10 (2004), 653.

TASCHNER: Privatrechtsentwicklung durch die Europäische Gemeinschaft Rechtsgrundlagen, Ziele, Sachgebiete, Verfahren, in: MÜLLER-GRAFF (Hrsg.), Gemeinsames Privatrecht in der Europäischen Gemeinschaft, 2. Aufl., Baden-Baden 1999, S. 225.

VAN OOYEN: Die Staatstheorie des Bundesverfassungsgerichts und Europa Von Solange über Maastricht zum EU Haftbefehl, Baden-Baden 2006.

VERVAELE: Mercosur and Regional Integration in South America, Int. Comp. Law. Q. 54 (2005), 387.

WEBER, ALFRED: Die Krise des modernen Staatsgedankens in Europa, Stuttgart 1925.

ZIMMERMANN: Konturen eines europäischen Vertragsrechts, JZ 1995, 477.

ZIMMERMANN: Die Principles of European Contract Law als Ausdruck und Gegenstand europäischer Rechtswissenschaft, Jura 2005, 289 ff. und 441 ff.

ZIMMERMANN: Die Unidroit-Grundregeln der internationalen Handelsverträge 2004 in vergleichender Perspektive, ZEuP 2005, 264.

ZÜRN: Regieren jenseits des Nationalstaates: Globalisierung und

Denationalisierung als Chance, 2. Aufl., Frankfurt/M. 2005. 